\title{
Investigating ultraluminous X-ray sources through their multiwavelength variability and broadband spectra
}

\author{
Luca Zampieri ${ }^{1}$, Elena Ambrosi ${ }^{2}$ and Amid Nayerhoda ${ }^{2,3}$ \\ ${ }^{1}$ INAF-Astronomical Observatory of Padova \\ Vicolo dell'Osservatorio 5, 35122 Padova, Italy \\ email: luca.zampieri@oapd.inaf.it \\ ${ }^{2}$ Department of Physics and Astronomy, University of Padova, \\ Vicolo Osservatorio 3, 35122 Padova, Italy \\ ${ }^{3}$ Institute of Nuclear Physics PAN, \\ ul. Radzikowskiego 152, 31-342 Kraków, Poland
}

\begin{abstract}
The population of ultraluminous X-ray sources encompasses accreting compact objects with significantly different masses, from black holes of intermediate mass, to black holes of stellar origin, to neutron stars. Investigating these sources will help us answering crucial questions on the distribution of black hole masses and on mass accretion above the Eddington limit in the local Universe, that have potential implications in other astrophysical areas. In order to perform a detailed investigation of ultraluminous X-ray sources, an accurate modeling of their evolution and multiwavelength emission properties is needed. We report some preliminary results of the activities that we are carrying out at present in this area.
\end{abstract}

Keywords. accretion, accretion disks, methods: numerical, X-rays: binaries

\section{Introduction}

Ultra-Luminous X-ray sources (ULXs) are point-like off-nuclear X-ray sources in nearby galaxies, intrinsically powerful but observationally rather faint. Their luminosity exceeds (although not necessarily all the time) the Eddington limit for spherical accretion onto a $\sim 10 M_{\odot}$ black hole $\left(L>10^{39} \mathrm{erg} \mathrm{s}^{-1}\right)$. Hundreds of sources have been discovered and reported in various surveys/catalogues to date. The observational evidence points towards the identification of the majority of ULXs $(\sim 60-70 \%)$ with accreting binaries (see e.g. Feng \& Soria 2011 for a review).

Two dynamical mass measurements of the compact objects in ULXs have been recently performed through dedicated spectral and/or photometric monitorings of their optical counterparts. M 101 ULX-1 has a black hole $(\mathrm{BH})$ with mass $M_{B H}>5 M_{\odot}$ (Liu et al. 2013), while the mass of the compact object in NGC $7793 \mathrm{P} 13$ is $<15 M_{\odot}$ (Motch et al. 2014). The latter system was very recently discovered to show X-ray pulsations and then hosts a neutron star (Fuerst et al. 2016, Israel et al. 2016a). Other two pulsating neutron stars (NSs) have been identified among ULXs (M 82 X-1, Bachetti et al. 2014; NGC 5907 ULX-1, Israel et al. 2016b), suggesting that pulsar ULXs may not be uncommon.

On the other hand, a few extremely luminous or hyperluminous sources, most notably HLX-1 in ESO 243-49 (with a peak luminosity of $10^{42} \mathrm{erg} \mathrm{s}^{-1}$; Farrell et al. 2009), have been identified as very good candidates intermediate mass BHs. For HLX-1, BH mass estimates based on X-ray spectroscopy and radio jet emission are in the ballpark of $10^{4}-10^{5} M_{\odot}$ (Servillat et al. 2011, Webb et al. 2012). 
These findings indicate that ULXs may contain compact objects of different types and that they may have a key role in exploring the origin and unknown distribution of $\mathrm{BH}$ masses in the local Universe. They could help us understanding what are the heaviest $\mathrm{BHs}$ that can form through direct collapse of massive stars, whether the BHs in ULXs may have something to do with the massive BHs recently discovered with the Laser Interferometer Gravitational-Wave Observatory, whether we can find further evidences of the existence of intermediate mass BHs, or what is the maximum luminosity and spin-up rate of magnetized NSs accreting above the Eddington limit.

\section{ULX binaries and accretion above the Eddington limit}

After the discovery of the gravitational wave event GW150914 with the Laser Interferometer Gravitational-Wave Observatory (Abbott et al. 2016), we know that massive $\left(\sim 30 M_{\odot}\right)$ BHs exist. As ULXs appear to host compact objects with significantly different masses (and origin), a natural question is whether BHs in a similar mass range could reside also in ULXs. A possible indirect evidence in favour of the existence of massive $\left(30-80 M_{\odot}\right)$ BHs in ULXs comes from their association with low metallicity environments (e.g. Mapelli et al. 2010), where they may form through the direct collapse of massive progenitor stars that have retained a significant fraction of their initial mass (Mapelli et al. 2009, Zampieri \& Roberts 2009, Belczynski et al. 2010).

To produce an active ULX phase, massive BHs and more conventional stellar-mass $\mathrm{BHs}$ have to cross a Roche-lobe-fed evolutionary phase. We investigated the evolution of $\mathrm{BH}$ binaries with massive companions in clusters including metallicity-dependent stellar evolution and found that dynamical interactions play a significant role (Mapelli \& Zampieri 2014, and references therein). As a consequence, both stellar-mass and massive BHs of stellar origin can power a non-negligible number of Roche-lobe-fed ULXs.

Besides the nature of their compact objects, the other challenge in understanding ULXs is the character of their accretion flow, which is able to produce luminosities 10-100 times larger than the Eddington limit. Even accounting for a reasonable beaming factor $(\geqslant 10 \%)$, the accretion rate needed to sustain these luminosities is larger than $10^{19} \mathrm{~g} \mathrm{~s}^{-1}$. The question is then what is the accretion physics for BHs or NSs emitting/accreting at this pace. In this respect, ULXs represent laboratories for extreme accretion environments (where photon advection/trapping becomes important), that may be relevant for the first generation of Quasars at very high redshift.

Supercritical accretion onto BHs has been studied by means of 2D magneto-hydro simulations. They show the formation of an advection-dominated disc and an outflow region, with powerful clumpy winds driven by radiation pressure (e.g. Takeuchi et al. 2013). This structure can explain some basic facts concerning the X-ray spectral components and the short-term variability at high energies observed in some ULXs (Middleton et al. 2015). This interpretation is supported also by the detection of emission lines and blueshifted (0.2c) absorption lines from highly ionized Fe, $\mathrm{O}$ and $\mathrm{Ne}$ in high-resolution X-ray spectra of NGC 1313 X-1 and NGC 5408 X-1, although the cumulative significance is only slightly above $5 \sigma$ (Pinto et al. 2016).

Supercritical accretion onto NSs is less studied. In order to account for the observed luminosity and long-term spin-up of the pulsar, the accretion rate and the magnetic field $B$ at the base of the accretion column have to be sufficiently high. However, the latter should not be too high to trigger the propeller stage and prevent accretion at the magnetospheric radius. These constraints give values of $B$ in the range $\sim 10^{12}-10^{14}$ $\mathrm{G}$ and suggest that the magnetic field may have multipolar components (Bachetti et al. 2014, Israel et al. 2016a,b). If the donor is more massive than the NS, a Roche-lobe-fed 

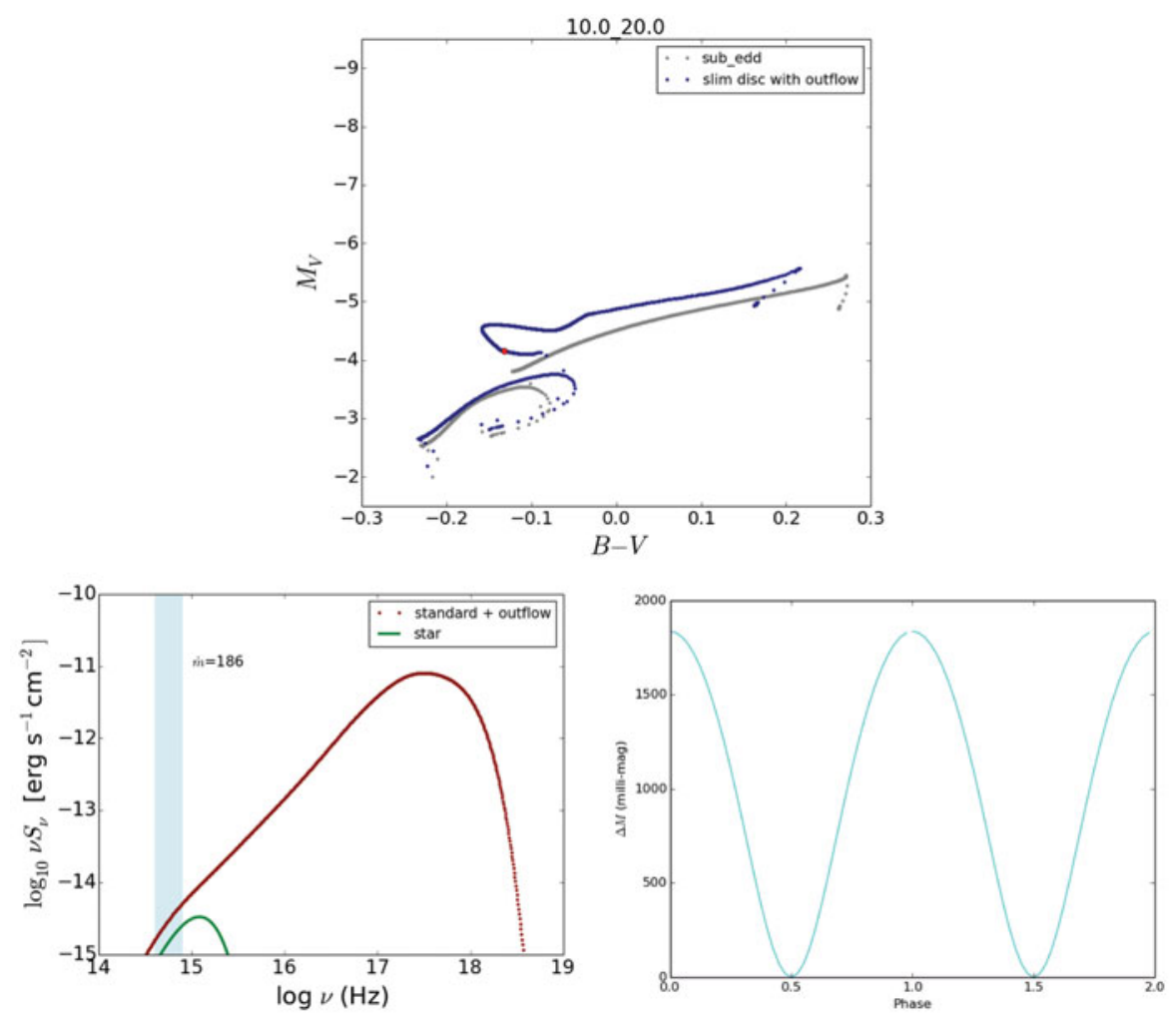

Figure 1. Top: Evolution on the colour-magnitude diagram of a ULX binary system with a BH mass $M_{B H}=20 M_{\odot}$ and a donor mass $M_{*}=10 M_{\odot}$ (at zero age main sequence). The lower track (gray dots) represents the evolution calculated assuming standard sub-Eddington accretion (Patruno \& Zampieri 2008). The upper track (blue dots) represents the new evolution computed for super-Eddington accretion. Bottom-left: Optical-through-X-ray spectrum of the system at the phase marked with a bigger $(r e d)$ dot on the evolutionary track for super-Eddington accretion $\left(\dot{m}=186\right.$ and the actual mass of the star is $\left.4.4 M_{\odot}\right)$. The upper $($ red $)$ points represent the spectrum of the self-irradiated outer disc plus the outflow and the innermost slim disc, while the lower (green) line is the spectrum of the X-ray heated donor. The vertical (light blue) strip marks the optical band. Bottom-right: Orbital modulation of the optical light curve caused by the irradiated donor for an inclination angle of $30^{\circ}$.

system is unstable. However, it may still be relatively long lived, if (thermal timescale) mass transfer is stabilized by stellar and disc winds/outflows (Fragos et al. 2015).

\section{A comprehensive multi-wavelength variability emission model}

Our goal is to investigate the emission properties at super-Eddington rates and use them to constrain the physical properties of ULXs. Thus, we developed a model to calculate the optical-through-X-ray emission of ULX binaries during their evolution, accounting for super-Eddington accretion (Ambrosi \& Zampieri, in preparation). The starting point is the model of Patruno \& Zampieri (2008), that includes X-ray irradiation. For accretion rates below the Eddington limit, the treatment of the accretion flow is that of Patruno \& Zampieri (2008). However, for accretion rates above Eddington, a bimodal structure is assumed: an inner flow with non-standard (slim) disc geometry and temperature profile (vertical scale height comparable to the radius and $T \propto r^{-1 / 2}$ ), and an outer flow with standard structure. The transition to the standard disc occurs where the inward 
advected heat is equal to the viscously dissipated heat. Following Poutanen et al. (2007), in our last implementation an optically thick outflow is also included in the treatment when $\dot{m}$ (the accretion rate in units of the Eddington rate) exceeds unity. The portion of the inner disc that illuminates the outer regions, where the UV-optical-IR radiation is produced, changes significantly from that considered in previous works.

Our model incorporates also a calculation of the expected orbital modulation of the optical light curve caused by the emission of the geometrically distorted and X-ray irradiated companion (Nayerhoda \& Zampieri, in preparation). The donor is assumed to fill or slightly underfill its Roche lobe. This calculation is being generalized at present to include the contribution of the optical emission produced by the irradiated outer disc.

The implementation of the model is in progress. In Figure 1 we show some preliminary results of the calculation of the expected emission properties for a ULX binary system with a $\mathrm{BH}$ mass $M_{B H}=20 M_{\odot}$ and a donor mass $M_{*}=10 M_{\odot}$ (at zero age main sequence). A snapshot of the optical-through-X-ray spectrum and of the optical light curve during a post-main sequence super-Eddington phase is also shown. Owing to the fact that the accretion rate is above the Eddington limit, in the post-main sequence phase the evolutionary track is brigther and bluer than that computed assuming Eddingtonlimited accretion.

The next step will be to test our model against ULXs with measured or constrained compact objects masses, as NGC 7793 P13, that contains a neutron star, is accreting above Eddington, and has detailed optical light curve and broadband spectral measurements available. To this end, we plan to inlude the emission from the accretion column of a magnetized neutron star to determine the X-ray continuum spectrum. The model will then be used for calculating the emission of self-consistent ULX binary system configurations computed following their evolution in clusters through N-body simulations (extending our previous work in this area; see Mapelli \& Zampieri 2014).

We acknowledge financial contribution from the grant ASI-INAF I/037/12/0.

\section{References}

Abbott, B. P., Abbott, R., Abbott, T. D., et al. 2014, PhRvL, 116, article id. 061102

Bachetti, M., Harrison, F. A., Walton, D. J., et al. 2014, Nature, 514, 202

Belczynski, K., Bulik, T., Fryer, et al. 2010, ApJ, 714, 1217

Farrell, S. A., Webb, N. A., Barret, D., et al. 2009, Nature, 460, 73

Feng, H. \& Soria, R. 2011, New Astronomy Reviews, 55, 166

Fragos, T., Linden, T., Kalogera, V., \& Sklias, P. 2015, ApJ, 802, L5

Fuerst, F., Walton, D. J., Stern, D., et al. 2016, ApJL, in press (arXiv:1610.00258)

Israel, G. L., Papitto, A., Esposito, P., et al. 2016, MNRAS Letters, in press (arXiv:1609.06538)

Israel, G. L., Belfiore, A., Stella, L., et al. 2016, ArXiv e-prints, (arXiv:1609.07375)

Liu J.-F., Bregman, J. N., Bai, Y., et al. 2013, Nature, 503, 500

Mapelli, M., Colpi, M., \& Zampieri, L. 2009, MNRAS, 395, L71

Mapelli, M., Ripamonti, E., Zampieri, L., Colpi, M., \& Bressan, A. 2010, MNRAS, 408, 234

Mapelli, M. \& Zampieri, L. 2014, ApJ, 794, article id. 7

Middleton, M. J., Heil, L., Pintore, F., et al. 2015, MNRAS, 447, 3243

Motch, C., Pakull, M. W., Soria, R., et al. 2014, Nature, 514, 198

Patruno, A. \& Zampieri, L. 2008, MNRAS, 386, 543

Pinto, C., Middleton, M. J., \& Fabian, A. C. 2016, Nature, 533, 64

Poutanen, J., Lipunova, G., Fabrika, S., et al. 2007, MNRAS, 377, 1187

Servillat, M., Farrell, S. A., Lin, D., et al. 1995, ApJ, 743, article id. 6

Takeuchi, S., Ohsuga, K., \& Mineshige, S. 1995, PASJ, 65, article id. 88

Webb, N., Cseh, D., Lenc, E., et al. 2012, Science, 337, 554

Zampieri, L. \& Roberts, T. P. 2009, MNRAS, 400, 677 